\title{
Perceptual Features for Off-line Handwritten Word Recognition: \\ A Framework for Heuristic Prediction, Representation and Matching
}

\author{
Sriganesh Madhvanath ${ }^{1}$ and Venu Govindaraju ${ }^{2}$ \\ 1 IBM Almaden Research Center, 650 Harry Road, \\ San Jose, CA 95120, USA \\ srigoalmaden.ibm.com \\ 2 Center of Excellence for Document Analysis and Recognition (CEDAR) \\ Department of Computer Science, State University of New York at Buffalo \\ Buffalo, NY 14260, USA \\ govindecedar buffalo. edu
}

\begin{abstract}
Perceptual holistic features are visually conspicuous features of the word shape that have been cited in reading studies as being utilized in fluent reading. While these features have been used for word recognition when the lexicon of possible words is small and static, their application to the general problem of omni-scriptor handwritten word recognition is limited by their variability at the word level and the paucity of samples for word-level training. A methodology of coarse holistic features and heuristic prediction of ideal features from ASCII is proposed to address these issues. The methodology is based on the view that realworld examples of handwritten words are instances of the ideal exemplar of the word class distorted by the scriptor, stylus, medium and intervening electronic imaging processes, and has applications in verification and lexicon reduction for handwritten word recognition.
\end{abstract}

Keywords: holistic approach, handwritten word recognition, perceptual features, graph-matching, handwriting models, syntactic approach

\section{Introduction}

Evidence from psychological studies of reading indicates that humans use perceptual features such as ascenders, descenders and word length in addition to letter identities in fluent reading [1]. Several of these features have found application in computer systems for recognition of handwritten words. Algorithms based on word-shape features are often said to be "holistic" in paradigm, as opposed to "analytical" approaches that determine the identity of the word from the identities of smaller units such as characters [2].

The majority of systems based on perceptual features have been designed for recognition problems involving a small, static lexicon of possible words, notably for legal amounts on checks [3-7]. The application of perceptual features to the 
general problem of off-line omni-scriptor handwritten word recognition where lexicons may be large or dyn-mically generated is limited by the variability of these features with respect to writing style and the paucity of samples for wordlevel training.

Word-level feature variability stems from the fact that every word in the lexicon is a pattern class from the standpoint of the holistic paradigm. Given any set of holistic features, a large degree of intra-class variation may be expected in the offline context wherein writing is unconstrained and images contain noise from the medium and surroundings. When the classes are numerous or dynamically determined, the separability of word classes in feature space is considerably diminished, and overwhelmed by intra-class variations. A small static lexicon permits the collection of training samples of each word class, and the use of statistical and syntactic methods for classification. Paucity of training samples refers to the fact that in the large/dynamic lexicon scenario, it is not possible to collect enough samples of each word to represent even the common writing styles. The large intra-class variation in handwritten text also rules out realistic simulation of offline handwriting samples for training.

In this paper, we describe a methodology of coarse perceptual features and heuristic prediction of ideal features of words, that allows the application of perceptual features to offline recognition scenarios involving large or dynamically generated lexicons.

\section{Methodology}

Real-world examples of a handwritten word may be modeled as distortions of an "ideal" word exemplar of the word class. The ideal exemplar is pure cursive, devoid of baseline skew and character slant, exhibits evenly spaced reference lines, and conforms approximately to the style adopted for writing instruction imparted in elementary school. The writing style adopted by an individual in adulthood may thus be regarded implicitly as a distortion of the cursive ideal learned as a child. In the absence of training samples and a handwriting model to synthesize them, features of the ideal word exemplar may be obtained directly from ASCII using a set of heuristic rules.

Recognition of real exemplars of a given word class as distortions of the ideal exemplar may be achieved by a combination of techniques designed to counteract or compensate for different forms of distortion. We propose the following hybrid approach to address the external influences that distort the ideal shape of a handwritten word : (i) preprocessing for slant normalization and noise removal (ii) angular or local reference lines for invariance with respect to baseline skew (iii) coarsely described perceptual features, and (iv) feature extraction algorithms, representations and matching algorithms that are robust with respect to residual slant, noise and fragmentation, and variations in stroke width and writing style.

We make several simplifying assumptions regarding the granularity of perceptual features. Perceptual features are classified broadly as scalar (those such 
as length that are completely specified by a single number) and positional (those such as ascenders which occur at different positions in the word). Values and positions are associated with scalar and positional features respectively, and confidences are associated with both kinds of features. Features extracted from an image of a handwritten word may differ from the ideal features of the corresponding word class in confidence and/or value (position), within certain bounds. In addition, positional features may have been missed during feature extraction, and spurious features may have been detected.

\subsection{Extraction of Perceptual Features from Images}

The framework for perceptual features described in this paper is independent of the actual perceptual features and feature extraction algorithms used. We describe in brief one method for extracting ascenders, descenders and length and one possible representation for values and positions.

Eight-connected components are detected in a right-to-left, top-to-bottom scan of the binary image and their contours traced to yield a list of chain coded exterior and interior contours. The mean character slant is computed from relatively vertical stretches of contour and the contours sheared and smoothed. Baseline skew is computed by linear regression over local minima extracted from the lower contour. Reference lines are determined from the angular histogram of contour crossings at the skew angle, which in turn is computed from a single traversal of the contours.

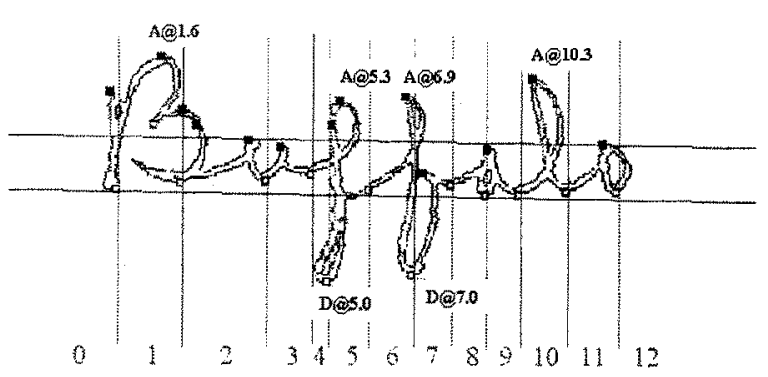

Fig. 1. Candidate ascenders and descenders in a word image and corresponding positions represented in Holistic Segment Distance (HSD)

Candidate ascenders and descenders are derived from local maxima on the upper contour and local minima on the lower contour respectively, as shown in Fig. 1. The significant local minima on the lower contour divide the image vertically into $n$ segments. Segments are numbered from 0 to $(n-1)$, and $n$ is used directly as an estimate of word length. Positions of features are specified in terms of Holistic Segment Distance (HSD). Each position is of the form $x . y$, where $x$ is the segment number, and $y$ is the offset into the segment computed 
as a fraction of the width of the segment. Segment distance preserves continuity of position across segment boundaries while affording greater precision than the segment number alone.

\subsection{Heuristic Prediction}

The expected holistic features of lexicon words are predicted from ASCII using a set of heuristic rules and models of "ideal" lower and upper case characters These models provide coarse descriptions of the shape of features in terms of the number of minima and positions of ascenders and descenders relative to the minima. In addition to the features that may be normally expected of a handwritten character, the character models also specify optional features of the character. Optional features allow modeling of alternate ways of writing the same character (for example, ' $G$ ' and ' $f$ ' may be written with or without descenders) and facilitate modeling of the additional features created when strokes fail to connect (for example, an additional ascender results when the two vertical strokes of an ' $A$ ' fail to touch at the apex). Finally, optional features allow compensation for spurious features resulting from fragmentation in the image, untidy writing, and inaccuracy of reference lines.

Given an ASCII string, the positions of ascenders and descenders are derived from the positions of these features of the constituent characters, as illustrated in Fig. 2, and are expressed in terms of the Holistic Segment Distance described earlier. The word length is computed as the sum of the ideal lengths of characters in the word. Derived features are assigned confidences of unity.

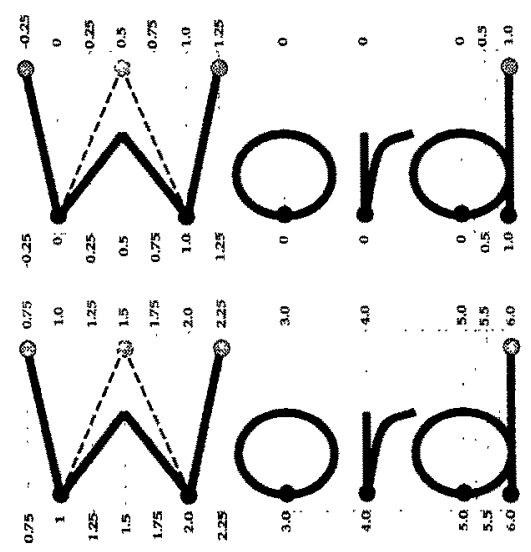

Fig. 2. Character models showing ideal positions of features in HSD. Optional features are shown by dotted lines. The positions of word features are derived by "concatenating" models of the constituent characters. 


\section{Modeling Distortion of Positional Features}

The positional features of an "ideally written word" occur in the ideal positions and have ideal confidences of unity. In practice, the same features are found to have shifted to the left or right (positional distortion) and differ in confidence (confidence distortion). For the purposes of modeling the distortion of positional features, positional and confidence distortion are assumed separable, i.e., the positional distortion $p f t(p, q)$ is assumed to be a function only of the feature positions, and the confidence distortion $c f i t(p, q)$ a function only of the confidences of features $p$ and $q$. The net goodness of matching $p$ with $q$ may be computed as $f i t(p, q)=p f i t(p, q) \times c f i t(p, q)$.

\subsection{Positional Distortion}

Two models of positional distortion, the Independent Position Model, and the Tied Position Model are proposed, both based on the underlying principle that ideal features have ideal positions from which they may shift in either direction within limits. Positional features are represented as solid spheres along a "groove" representing the length of the word. Since the object is to model positional distortion exclusively, the spheres are only allowed one degree of freedom - they may move to the left or the right along the groove within limits, and may not cross one another or move beyond the ends of the word.

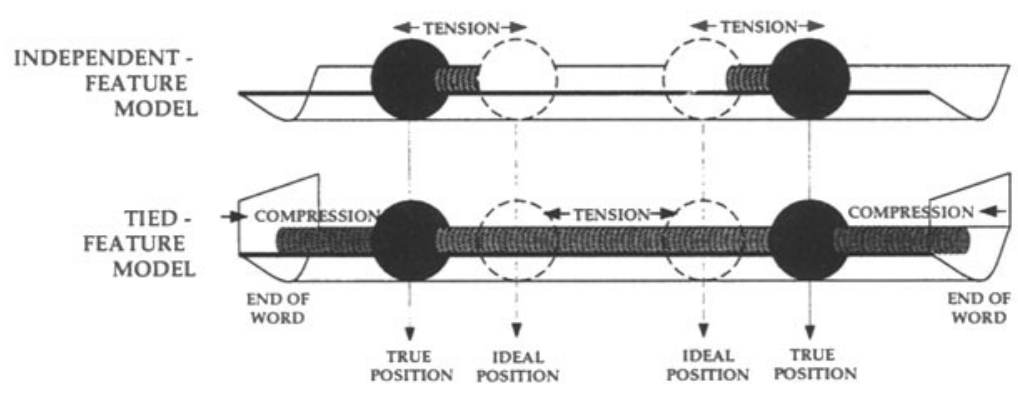

Fig. 3. Two models of positional distortion

In the independent-feature model, distortion of individual holistic features are assumed to be independent of one another. The spheres are connected to their ideal positions along the word by tightly coiled springs (Fig. 3(a)). In the tiedfeature model on the other hand, features are tied to one another and to the ends of the word by springs, and consequently their distortions are not independent (Fig. 3(b)).

The positional distortion incurred in matching a set of image features with the ideal features is measured in terms of the sum total of the work done in stretching or compressing the springs. Only tensile forces occur in the independent-feature 
model, while both tensile and compressive forces occur in the tied-feature model. The work done in stretching or compressing a spring is related to the square of the stretch $s$ by the equation $F=k \cdot s^{2}$, where $k$ is the spring modulus and a measure of stiffness of the spring. The spring modulus is a function of, among other things, the unstretched length of the spring, which in turn may be modeled by the distance between the ideal feature positions. In practice, one or both models are implemented implicitly by the function $p f i t(p, q)$ which measures the degree of positional distortion between two given positional features $p$ and $q$.

\subsection{Confidence Distortion}

Positional features such as ascenders, descenders and loops have an alternative interpretation as pattern classes. The manifestations of intended ascenders in real samples of handwriting together constitute the pattern class 'Ascender'. The confidence attribute associated with a candidate ascender could reflect the probahility that the feature is a member of the pattern class 'Ascender'. An alternative interpretation based on Fuzzy Set Theory is that of membership in the fuzzy set 'Ascender'. In general, any reasonable measure of distance may be used to model confidence distortion.

\section{Matching of Perceptual Features}

We have proposed a graph-based formalism for representation and matching of the perceptual features of the image and the lexicon entry [8]. The image is represented by a wordgraph, which is a set of feature nodes. A feature node in turn, is an abstract representation of a perceptual feature, and has three attributes: type (type of feature), value (value for scalar feature, and position for positional feature) and confidence (assigned by feature extraction to reflect the confidence that the feature exists at the said position or has the said value).

The matching scheme allows a feature of one word to match a feature of another if they are of the same type, and are at approximately the same position or have approximately the same value, independent of other extracted features. The specification of maximum allowable drift serves to prune the possible associations between features of the same type to a reasonable number, regardless of which of the positional distortion models is employed. Matching involves finding the best bipartite match between these two sets of nodes using the functions $c f i t()$ and $p f i t()$, subject to the constraint that the ordering of positional nodes of a particular type be preserved in the match. Details of the formalism and matching algorithm may be found in [8].

\section{Applications}

Perceptual features provide a coarse characterization of word shape, and are clearly insufficient to make fine distinctions between word classes. However, they 
are useful in distinguishing word classes with clearly dissimilar coarse shapes, and consequently usefu' in the verification of handwritten words and phrases and reduction of lexicons.

Word verification refers to the task of deciding whether or not a given ASCII string is the truth of a given binary image of a handwritten word and is useful in scenarios when a word image has been classified but the classifier is unsure of its result. In [9], we describe a system for rapid holistic verification of unconstrained handwritten phrases, based on the framework for representation and matching of perceptual features developed in this paper.

We have also applied this framework to the task of lexicon reduction, the rapid elimination of unlikely lexicon candidates prior to recognition [8]. The system described in this paper is used to reduce dynamically generated lexicons given isolated handwritten words in cursive, discrete and mixed writing styles.

\section{Summary}

'Iolistic methods based on perceptual features developed for small, static lexicons are not easily extended to large and dynamic lexicons owing to word-level feature variability and paucity of training samples. A methodology of coarsely described perceptual features and heuristic prediction of ideal features from ASCII is proposed to address these issues. The proposed methodology is based on the axiom that real-world examples of handwritten words may be viewed as the ideal exemplar of the word class distorted by the scriptor, stylus, medium and intervening electronic imaging processes. Perceptual features of images are viewed as distortions in position and confidence of the ideal features of the corresponding ideal exemplar. Perceptual features of images as well as lexicon entries are represented uniformly by wordgraphs, and are matched using a constrained bipartite graph matching algorithm. While insufficient to make fine distinctions between word classes, the coarse characterization of perceptual features is sufficient for detection of dissimilarities in word shape. This observation forms the basis for the proposed applications of word verification and lexicon reduction.

\section{References}

1. Soltysiak, S.J.: Visual information in word recognition: Word shape or letter identities? In: Proc. Wkshp on Integration of Nat. Lang. and Vision Proc,, Seattle, USA, Aug. 2-3 (1994)

2. Lecolinet, E., Baret, O.: Cursive word recognition; Methods and strategies. In: Impedovo, S. (ed.): Fundamentals in Handwriting Recognition. Springer-Verlag (1993) 235-263

3. Paquet, T., Lecourtier, Y.: Handwriting recognition: Application to bank cheques. In: Proc. First Intl. Conf. Document Anal. Recog., Saint-Malo, France, Sept. (1991) 749-757

4. Moreau, J.V.: A new system for automatic reading of postal checks. In: Impedovo, S., Simon, J.C. (eds): From Pixels to Features III: Frontiers in Handwriting Recognition. North-Holland (1992) 171-184 
5. Simon, J.C., Baret, O., Gorski, N.D.: A system for the recognition of handwritten literal amounts of checks. In: Spitz, A.L., Dengel, A. (eds): Proc. IAPR Wkshp Document Anal. Sys. World Scientific (1994) 265-287

6. Guillevic, D., Suen, C.Y.: Cursive script recognition applied to the processing of bank cheques. In: Proc. Third Intl. Conf. Document Anal. Recog., Montreal, Canada, Aug. 14-16. IEEE Computer Society (1995) 11-14

7. Olivier, C., Paquet, T., Avila, M., Lecourtier, Y.: Recognition of handwritten words using stochastic models. In: Proc. Third Intl. Conf. Document Anal. Recog., Montreal, Canada, Aug. 14-16. IEEE Computer Society (1995) 19-23

8. Madhvanath, S., Govindaraju, V.: Holistic lexicon reduction. In: Proc. Third Intl. Wkshp. Frontiers in Handwriting Recog., Buffalo, USA, May 25-27 (1993) 71-81

9. Madhvanath, S., Kleinberg, E., Govindaraju, V., Srihari, S.N.: The HOVER system for rapid holistic verification of off-line handwritten phrases. In: Proc. Fourth Intl. Conf. Document Anal. Recog., Ulm, Germany, Aug. 18-20. IEEE Computer Society (1997) 855-859 